\title{
The Application Value of Virtual Reality Technology on Visual Transmission
}

\author{
Chen Ping \\ Guagdong University of Science \& Technology \\ Dongguan, Guangdong, China
}

\begin{abstract}
Virtual reality technology is the Chinese translation of English Virtual Reality, referred to as VR, refers to auxiliary devices such as docking control devices, processing and computing information through computer programs, and finally interacting, simulating natural state and three-dimensional environment formed in the display terminal. A technique that creates immersion. The application of virtual reality technology to communication activities has produced virtual reality communication; the application of virtual reality technology to news reports has spawned the emergence of virtual reality news. The great significance of virtual reality technology for news dissemination lies in: comprehensively ingesting, recording, and transmitting news material, and letting news events "reproduce" and "restore". Virtual reality technology can realistically reproduce the scene of the real scene and set the action of the target by simulating the real scene, so that the user can clearly understand the scene. This paper discusses the characteristics and applications of virtual reality technology, focusing on the application value of virtual reality technology in the visual direction.
\end{abstract}

Keywords—visual reality; visual transmission; application value

\section{INTRODUCTION}

At the 2013 China Virtual Reality Industry Summit, Huai Guomo, deputy director of the former Commission of Science, Technology and Industry for National Defense, said that the research on the virtual reality industry, from the early days of the 21 st century, to the rapid development of the country, first benefited from The progress of virtual reality technology itself shows a strong media advantage; secondly, people have a new pursuit of the way and platform of information exchange. When General Secretary $\mathrm{Xi}$ Jinping participated in the investigation and study of Zhongguancun in Beijing, he also believed that science and technology is the backbone of the development of material society. Since 2004, China has held a virtual reality industry summit every year. However, due to the immature technology development, the development of other industries such as the media industry has gradually ushered in the peak of the Internet era. Virtual reality has received little attention and attention from the industry. . However, today, with the continuous development and innovation of technology, virtual reality technology is appearing in front of us in a swift manner. When such a new thing comes to us, we also need to get close to it, understand it, use it, and virtual reality technology is constantly integrated with the Internet and selfmedia. It must be like the iPhone changed the smart phone and extended to the future. Every aspect of our lives in the Internet age. The most striking feature of virtual reality technology in practical use is immersion. This is to promote the landscape communication of existing texts, images, videos, etc. in the field of communication to the spectacle and immersive experience of "experience". This is undoubtedly a highly disruptive technological change, and it is likely that it will directly lead to the transformation of the existing state of many industries.

\section{ViRTUAL REALITY TECHNOLOGY FEATURES}

Virtual reality technology is a visual effect technology that uses computer simulation to simulate events that have occurred, events that are running, events that are expected to occur, and so on. Virtual reality technology has a large adjustable type, which can gradually complete the events in a series of virtual scenes according to people's prior knowledge points, so that the audience feels real and has certain reliability. Virtual reality technology has been widely used in a series of high-tech applications such as military, traffic sector accident playback, locomotive operation monitoring, etc. Therefore, virtual reality technology will have great application prospects for a long time in the future. The three main elements of virtual reality technology are "immersion", "interaction" and "conception". For "immersion", the purpose of "immersion" is to make users as much as possible immersed in the three-dimensional scene simulated by virtual reality technology, so that users have an immersive feeling, increase the authenticity of virtual reality scenes, and thus improve virtuality. The quality of the real scene. "Interaction" refers to the virtual reality technology applied to the constructed virtual scene, with controllability, such as the passage of trains on high-speed railways, the switching of tracks, the speed of operation, the choice of running lines, and so on. For example, the MATALB software now provides a simulation of vrworld. The object of the scene passes through the virtual entity of the 3D software component to control the motion of the object of the virtual scene through programming. Therefore, the "interaction" feature has attracted more and more attention. The "conception" can sometimes be used, sometimes it is not needed. For the reproduction of the accident scene, the user only needs to know the passage of the incident, so it may not need to "conceive", but in the military field, the soldiers need to pass. Conduct combat drills on different terrains, conceive obstacles, etc., and create more realistic scenes to improve the level of simulation exercises. In short, virtual reality technology uses advanced computer technology (existing professional software) to make some 
scenes or processes in reality into an objective virtual environment by using high-tech technology to give people a feeling of touch, hearing and visual. Therefore, when users are applying the virtual reality technology, they often generate some consciousness or illusion similar to the real world, allowing the user to truly understand the virtual world's context and things from the senses such as smell and touch, so that people are immersed in In the virtual situation. Virtual reality technology is the crystallization of high technology, which makes the application of computer graphics more operability. Users can perform related rendering according to their needs. Virtual reality technology includes a series of artificial intelligence such as graphics, image processing, network technology and pattern recognition. Technology, combined with meteorological, geological, psychological, and aesthetic perspectives, uses a series of intelligent methods to make virtual reality scenes more attractive to the audience.

The field of virtual reality technology is extremely extensive. It is applied in the field of computer communication in BCGcentrex, the business system of the centrex, the data network of the IP network, the call center, etc., so that users can logically establish the acquisition of business data, using virtual memory and The communication of the virtual host completes the application of virtual reality technology. For example, the virtual call center provides a personalized service platform for enterprises and customers, which can effectively improve communication and information exchange services between customers and enterprises, and provide more professional services. Virtual reality technology enables highlevel integration of modeling technology and viewpoint control in the visual simulation process. It can be modeled by $3 \mathrm{D}$ through existing 3D modeling tools such as Maya, SoftimageXSI, Houdini, 3DS max, Lightware 3D and MUltigen Creator. The software and the 3D software bring the physical scene in the toolkit, the user can realize the rapid modeling and simulation design, and modify the texture features to derive the corresponding development model. 3D modeling includes geometric modeling and image modeling. Geometric modeling is a large part of the entire virtual reality scene. For large virtual scene rendering, the Murtigen Creator software can be used to quickly visualize virtual reality and obtain a geometric model that is very close to the actual physical model. Image modeling is based on geometric models, including related features such as texture, material, and illumination of geometric models. It is also an important part of virtual reality technology application. Virtual reality technology realistically reproduces actual scenes. The direction that draws the attention of the audience to achieve a satisfactory result. For image modeling, there are four main texture modification methods: projection texture, stereo texture, sphere texture and cylinder texture. Projection texture is the most widely used in virtual reality scenes. Projection texture can satisfy multiple angles. Rendering.

\section{HidDEN DANGERS OF VIRTUAL REALITY TECHNOLOGY IN COMMUNICATION}

For the time being, the hardware and software technologies of virtual reality technology are still in an unstable development. The production process lacks security and stability, and there is no compatibility between them. These defects are not suitable for VR news. Regular production. In the configuration of personnel, it is necessary to introduce and train special technical operators. Horizontal comparison of unmanned aircraft, now due to the widespread application of this technology, the social problems are also endless, the government is also studying the relevant policies of governance. In this way, since the production of virtual reality news also has specific requirements, it requires both technical debugging personnel and journalists, and requires close communication and cooperation between the two parties. On the other hand, the investment in large amounts of resources required for highprecision, interactive news increases production costs. Therefore, high-end VR news products are more expensive to produce, higher in technology, and longer in cycle. Therefore, the virtual reality news will be more biased towards the prolific production of the theme in the selection of the theme, mainly documentary and major theme news, such as the example of Syrian cultural relic protection mentioned above. In contrast, some small VR news works are difficult to visualize.

In addition to technical limitations, how to combine VR technology with existing news narrative techniques to tell stories is still a problem faced by practitioners. VR technology is to restore the real site, and provide interactive hardware support, lack of specific interaction of "role" in the "story", which is out of the scope of traditional photographic camera technology, similar to Traditional photography techniques such as montages have been unable to meet this requirement. From the perspective of traditional news reports, the main body of the report is the journalist, and VR can realize the "human"ization in terms of its own news reporting ability. In other words, virtual reality already has all the on-the-spot news material. He is like a department store. The audience is the customer and what is needed. In the traditional sense, the reporter analyzes the news scene through his own perspective. The news I saw was after the reporter's thinking and processed news. It is from this kind of thinking that it is more worthwhile to ask the question of whether virtual reality news needs journalists. The original intention of VR news products was to shorten the distance between the audience and the news, to change the acceptance mode of traditional users, and to turn the simple audience into the first witness of the "live". With the rapid development of virtual reality technology, various VRderived products in the market are emerging one after another. Under the background of the "homogeneous" development in the future, will it still be as attractive as it is now? When people have long been eccentric about this, can real news really attract them? This is also a problem. However, there is no shortcut to coping with technical challenges. The only solution is that news organizations must strengthen communication with technology companies, clarify the specific requirements and problems of both parties, work together, sum up experience in mistakes, keep up with the trend of the times and the needs of the masses. Improve the service quality of products, and carry 
out strict checks to form a good supervision atmosphere in the industry norms. In this way, we can better attract the public, expand market demand, and achieve healthy and stable sustained growth. In this process, news organizations and VR technology companies must maintain close communication. Otherwise, when capital flows into other more optimistic areas or markets, it may be too late, because the lack of integration is not the ultimate goal.

The media can reorganize the body's perception and consciousness. When a person wears a virtual reality device on his head, the various sensory organs of the head are wrapped up, and the brain receives the impact of information. The perceived content comes from this narrow "box" full of technology, which is easy to associate with Sheila. Ri Putnam's hypothesis of "the brain in the tank." He believes: "All things that I regard as the most authentic and reliable are accepted through the senses; but sometimes, there is evidence that our senses are deceptive." 1 Through this passage we can easily find The signals that our senses can feel and receive are deceptive, and we have no way to distinguish whether they actually exist. This can not help but remind people of the "Martian invasion of the earth" radio drama launched by Columbia in the 1930s caused a sensation in the United States. From the point of view, because it was too formal and true, people believed in this extremely absurd event and caused great panic to the society. After 20 to 30 years, the emergence and development of television has greatly promoted the hypnotic effect of modern media on people. In the era of big data, information and communication technology has been rapidly promoted under the rapid development of social media, forming a "information mortuary" in the communication between people. At the present VR, it can be said that human dependence on the media has been constantly increasing. This strong dependence on the media has gradually made us passive. The emergence of smart mobile devices such as smartphones and tablets has made us more and more unable to leave them. If we don't have them, people will become insecure. Under such circumstances, we have often lost the initiative, but we are hampered by these inanimate objects. For virtual reality, the changes it can bring will only be more than that for mobile phones. Will its strong effect make us human beings unable to fight? In summary, the arrival of this terrible scene of "the brain in the cylinder" is by no means nonsense. The rapid development of the media, while benefiting the society, should also trigger our vigilance and reflection.

In the first issue of "Weekend VR People", the researchers once thought about the topic of VR viewing and VR dining social networking. Although the technical difficulty does not seem high, the appeal to the audience seems to be far more than the traditional one. Similar social mode. However, how many people can understand the reasons for this motivation? For many researchers, the reason for this "similar person" VR experience is that the root cause is that they are blatantly invading the "safe distance" of real social behavior, whether it is close at hand. Watching a movie is still a diet on the same table (and there is no lack of communication, although it is a unilateral interaction), which is essentially an act that can be achieved under the premise of a more intimate relationship. However, given the core selling point of "deception", a contemporary civilian VR device, the user wearing the head display has the illusion of "enjoying the unrealistic experience in reality"; and this kind of "infringement of common sense" is harvested. Fun is the foundation for the realization of the above two ideas. With the basic theory, it will be slightly diverged: How many of the "Personal Life Records" that appear on many live broadcast platforms today can become a VR model, and how many audiences can enjoy it? The answer seems to be amazing - all. In fact, the closer to the live content of daily life, the more people can have the pleasure of "openly peeking into the lives of others". In a sense, this fascinating mechanism of pleasure formation can indeed remind us of some psychopathic behaviors that are close to mental illness. However, the Internet has used anonymity to expose these hypocrites to the true dark side of the heart, to see those who are addicted to the real life window to peer into the audience, how they will choose after the arrival of the VR era, presumably there is no need to say that everyone Will be clear.

\section{COUNTERMEASURES TO PREVENT HidDEN DANGERS OF VIRTUAL REALITY}

From a macro perspective, we must make overall planning for the development of the virtual reality industry, formulate a forward-looking industrial development plan, and design a road map for virtual reality and mutual integration and common development in various fields. Industry authorities should fully realize that virtual reality technology can bring leap-forward progress and even integration to all walks of life. Then, provide industrial policy support, clarify the ideas of industrial development, and guide them from policies, while integrating resources and exerting The revolutionary role of this technology in various industries enables industries to integrate virtual reality technology and innovate in industry technology. In the end, we will achieve leapfrogging in various industries. Under the integration of virtual reality technology and journalism, it can also provide more macro ideas and solve the universal problems in virtual reality news communication. In addition, relevant departments should establish relevant technical standards for the virtual reality industry as soon as possible and improve the technical system. The establishment of relevant technical standards will help to consolidate the layout of independent technology, establish a national independent brand, and improve the international discourse status of China's virtual reality industry. Only by establishing standards can we deliver accurate and high-end product concepts to consumers, avoiding consumers being deceived by some quick-minded manufacturers and choosing low-quality products, thus ensuring the healthy development of the virtual reality industry and promoting market progress and product consumption. By putting forward specific standards for equipment production, the state can control the specific technical indicators such as the perspective and response time of virtual reality products, not only to protect consumers' personal safety and physical and mental health, but also to provide consumers with better products. Experience the effect, and ultimately promote the benign competition in the industry and form a better benign human-computer interaction. In the virtual reality environment, a relatively closed interactive virtual environment is created for the individual audience. The information received is huge and complicated, and it is difficult 
to supervise. Therefore, the state should not only consider the safety of production and market, but also protect it. Citizens' information security, strengthen the management of information data, and strengthen the supervision of information dissemination channels. The explosive growth of virtual reality products will inevitably lead to a burst of data volume, so that a large amount of data, especially those related to the private life of citizens, will lead to serious consequences. Therefore, the development of product standards is not only for the production process, but also for data collection, data flow and scope of use. Broadly speaking, not only virtual reality products, but also information technology products that will appear in the future and in the future should be applied to this standard. Only by providing detailed descriptions of data collection and data usage of virtual reality products, and establishing a data management system, can ensure the safe and efficient use of data, ensure that citizens and industrial applications, and even state secrets are not leaked, and at the same time spread the efficiency of information. Constantly upgrade to new heights.

Virtual reality is currently facing the "upstream" overheating, "downstream" is too cold, the concept is being fired very hot, but most users only stay at the level of early experience, and rarely can continue to repetitively consume, which results in Most companies are still holding a wait-andsee attitude and are faltering into the virtual reality market. Virtual reality technology is still an emerging concept. It is difficult to achieve industrialization mainly relying on the involvement of venture capital. The special support of the state finance can promote the virtual reality industry to become bigger and stronger, integrate technology, products and market resources, and make virtual reality technology. It can support the development of key industries such as industry, culture and education, and finally integrate into national planning. Therefore, the practitioners of the virtual reality industry should also be more innovative with the support of the state financial support, research technical solutions and advance the experimental progress; more cooperative spirit, strengthen the coordination and collaboration between the upstream and downstream of the virtual reality industry chain, Constantly enhance product confidence and ability to self-confidence, dare to innovate, be good at innovation, play the "artisan" spirit; more spirit of struggle, dare to compete with the international, and consolidate independent technology on the basis of establishing and improving relevant technical standards and systems of the virtual reality industry. Layout, establish an independent and controllable industrial chain, promote the industrialization of virtual reality technology, and finally build an industrial platform that integrates many development advantages.

It is undeniable that virtual reality will generate huge consumer demand in the process of integration with various industries. However, the spread and spread of bad ideas and information, such as decay and backwardness, which are accompanied by it, are also one of the problems that need to be solved. Similar to cyberspace, in the virtual reality space, China should also formulate corresponding laws and regulations as soon as possible, improve the virtual reality space culture market, promote social melody, spread mainstream values, and vigorously support healthy, positive and optimistic cultural products, and advocate correctness. Harmonious and healthy cultural activities have enabled the party and the government to form a stronger appeal and influence in the virtual reality space. Due to the immersive viewing of virtual reality culture works, the appeal and authenticity of traditional culture can be deepened, making it an important propaganda window for Chinese culture to spread overseas. The management of related enterprises is also conducive to improving the industrial development environment. In the past, the traditional media has inspired us that high-quality brands are often directly proportional to quality content. China's quality supervision departments should adapt to the new situation and new development of industrial development, research and develop the evaluation model applicable to this technology, and grasp the quality evaluation work of virtual reality products through quality sampling and market supervision. Strive for a fair and just market environment. The healthy development of the industry is also inseparable from the brand building of key products. Through the strong support of excellent independent brands, it can cultivate relevant leading enterprises, and then lead the healthy and orderly development of the whole industry with leading enterprises in order to improve consumers' virtual reality. The degree of industry recognition, creating high-quality content, content as the core of the media industry, is also a strong support for building brands. In order to achieve this goal, the construction of key product brands can set up committees composed of experts in various fields in the industry to do a good job in policy consulting, industrial planning, technical support, marketing strategies and other aspects of services. At the same time, enterprises themselves should pay attention to the research of intellectual property rights. With the support of the state, they should look to the world and create high-level patents and ace IP.

The healthy development of an industry is inseparable from the coordinated development within the industry. This coordinated development can be achieved through an advisory committee composed of demonstration parks and experts in related fields, integrating technology innovation and research, product manufacturing and distribution, equipment application and promotion. Establish industry associations or enterprise alliances, and maintain close communication with the research institutes in the early stage of the research, update the emerging technology solutions and industrial development trends at home and abroad in real time, establish an information platform that is openly shared within the industry, and conduct industry through statistics of big data. Monitoring and analysis, providing information for the sustainable development of the industry, and playing the role of the platform in public services such as data statistics and information collection. At the same time, the information platform should also do a good job in quality supervision of virtual reality products, realize online publication of product quality information and enterprise credit information, and be open, fair and transparent. The platform can blacklist the problem enterprises and expose them on the Internet. At the same time, quality and safety warnings are issued to the production enterprises to strengthen product quality supervision and guide enterprises to pay attention to quality and safety construction. At the same time, enterprises 
should be good at absorbing innovative talents. They can explore talents through the Virtual Reality Innovation and Entrepreneurship Competition and provide sufficient professional talent support for the sustainable development of the industry. In the case of virtual reality news, in the process of virtual reality communication, relatively isolated "people" (present users) is the main body of information collection in the virtual environment, but does not mean that everyone has professional news literacy. Although the traditional journalist's perspective has certain subjective limitations, such professionals engaged in news gathering provide a "threshold" for news to think about and think about news events, and play a certain standard in the audience's understanding of news. The role, which is often referred to as the "gatekeeper" in the theory of communication. From the perspective of "cold and hot" relationship, what is generated between VR and the audience is a "hot" relationship of "distance". Then professional journalism literacy provides people with a kind of "keep distance". A "cold" relationship between reason and thinking, which makes the professional perspective and professional talents are particularly important under the strong effect of the VR environment. Virtual reality technology can only be used as a communication tool and allows technology and tools to serve news. In the process of realizing VR+ news, adhering to the professional news attitude and consistently holding on to the essence of the news, it is becoming more and more precious in the current pan-entertainment trend of mass media communication, and it is also a long-term solution for brand building.

\section{CONCLUSION}

Virtual reality technology is widely used in various industries, such as automobile operation, train transportation, accident scene simulation and other fields of reproduction and simulation. Virtual reality technology is reconstructed by 3D model and texture rendering makes the 3D model more suitable for the viewer's visual experience. Thereby creating a realistic scene. The immersive nature of virtual reality technology in the process of communication has created a fantastic way of communication, namely immersive communication. Immersive communication, a new type of communication, is gradually infiltrating into all aspects of our daily lives and social life, and changing our inherent cognitive styles and modes of thinking. We should and must regard this incredible new medium as a historical necessity in the development of human communication and communication activities. It is a question that needs careful consideration and repeated consideration, but it is undeniable that virtual reality technology has subverted humanity since the primitive period. The way of survival and experience of accumulation.

\section{REFERENCES}

[1] Writer's Handbook. Mill Valley, CA: University Science, 1989.

[2] Yan Lin. Application Value of Virtual Reality Technology in Visual Communication Direction[J]. Computer CD-ROM Software and Application, 2013(23):95-96.

[3] Gu Yue, Wu Youwei. Application Research of Virtual Reality Technology in Sports Events Broadcasting[J]. Modern Vocational Education, 2016(19):13-17.

[4] Wang Yuxin. Application Research of Virtual Reality Technology in TV Program Production[J]. Science \& Technology Communication, 2017(6)

[5] Ding Lei. Discussion on the Application of VR Virtual Reality Technology in TV Programs[J]. China Media Technology, 2017.

[6] Du Zhijun. Research on face expression synthesis technology[J]. 2010

[7] Zhang Xianzhi. Development and Application Research of Virtual Reality Technology [J]. Science and Technology Information, 2018,505(04):34+41.

[8] Li Wei. Application and Enlightenment of Information Technology in Visual Communication Design Teaching[J]. Art Science, 2017(2).

[9] Zhang Yiqi. Development and Application of Virtual Reality Technology [J]. Communication World, 2018, 340(09): 253-254

[10] Huang Ruojun. The Impact of Virtual Reality Technology on Advertising Communication - Taking the Application of VR Technology in Marketing as an Example[J]. Journalism Front, 2016(24):66-67. 